\title{
ALGUNOS CONCEPTOS MATEMÁTICOS ASOCIADOS AL INFINITO
}

\author{
Iván Darío Buitrago C. \\ Universidad de San Buenaventura \\ seccional Medellín \\ idbuitragoc@outlook.es
}

\author{
Carlos A. Gaviria \\ Universidad de San Buenaventura \\ seccional Medellín \\ carlos.gaviria@usbmed.edu.co
}

\author{
Carlos A. Márquez \\ Universidad de San Buenaventura \\ seccional Medellín \\ kamarfer@gmail.com
}

(Tipo de Artículo: Reflexión. Recibido el 20/11/2014. Aprobado el 12/12/2014)

\section{RESUMEN}

En éste artículo se aborda el concepto de infinito en su forma general y se hace un análisis del infinito actual y del infinito potencial. Se realizan algunas demostraciones, con el objetivo de enfatizar en la trascendencia que dejó la idea del infinito desde los griegos hasta nuestros días y lo alejado que está el concepto del sentido común. Se trabaja la idea del infinito actual, desde la teoría de conjuntos, tratada por los gran Bernhard Bolzano y Georg Cantor.

Palabras clave. Cálculo, conjuntos, derivada, infinito, infinitesimal, límites, matemáticas.

\section{SOME MATHEMATICAL CONCEPTS RELATED TO INFINITY}

\begin{abstract}
This article deals with the concept of infinity in its general form and an analysis about actual infinity and potential infinity is performed. Two demonstrations are performed, the Archimedean property and an inequality by means of mathematical induction. The aim of these two demonstrations is to emphasize on the significance that Greeks' idea of infinity have until nowadays and how far is this concept from common sense. The idea of actual infinity is examined based on set theory.
\end{abstract}

Keywords. Calculus, Sets, Differentiation, Infinity, Infinitesimal, Limits, Mathematics

\section{Quelques concepts mathématiques liés à l'infini}

\section{Résumé}

Cet article aborde le concept d'infini dans son forme général et réalise une analyse de l'infini actuel et l'infini potentiel. On présente quelques démonstrations, avec l'objectif de mettre l'accent sur l'importance de l'idée de l'infini depuis les grecs jusqu'à nous jours et son éloignement du sans commun. On travaille l'idée de l'infini actuel, d'après la théorie des ensembles, abordée par Bernhard Bolzano et Georg Cantor.

Mots-clés. Calcul, ensembles, dérivée, infini, infinitésimal, limites, mathématiques. 


\section{INTRODUCCIÓN}

El infinito es uno de los conceptos más importantes en las matemática el infinito es la matemática misma. Un concepto que atraviesa el cálculo diferencial, el análisis matemático, la teoría de conjuntos. Fue abordado por los pensadores más grandes que ha dado la humanidad, Aristóteles, Galileo, Newton, Leibniz, Cauchy, Giordano Bruno, Bolzano, George Cantor, Hilbert, y Borges, por nombrar sólo algunos.

\section{EL INFINITO}

El infinito, un concepto que no fue aceptado por Aristóteles, que llevó a la locura a Cantor, que empujó a la hoguera a Giordano Bruno, que enredó los pensamientos de Galileo, que generó protestas en Gauss, que rechazó Cauchy, que inspiró a Newton, que sobresaltó a Leibniz, que generó las paradojas de Bolzano, que engrandeció el espíritu Hilbert y que inspiró un cuento en Borges.

Las ideas brillantes normalmente van en contravía del sentido común, es tal vez por éste motivo que son difíciles de entender, y por qué no decirlo, algunas veces parecen imposibles de ser asimiladas por la razón. Este es el motivo por el cual deben existir seres que razonan con una lógica diferente, con una lucidez capaz de asombrar al más distraído de los seres humanos. Pensadores diferentes, capaces de perder la razón por una idea, el infinito.

¿Qué es la matemática sin el infinito? Nada. La matemática es entonces, definiciones, axiomas, teoremas, símbolos, pero la esencia misma de la matemática, el corazón, es la comprensión de algo que parece incomprensible, el infinito. Este concepto ha sido la piedra angular de conceptos claves como límite, derivada, sucesiones y series. José Ramón Ortiz, en su artículo "El concepto de infinito" asegura que:

"uno de los logros más grandes de la matemática como lenguaje ha sido su propio coraje imaginativo para enfrentar el concepto más inaccesible que haya podido pretender la fragilidad temporal del intelecto humano: el concepto de infinito. Casi podríamos decir que la matemática es el lenguaje que pretende medir el infinito" [1].

Por su parte Fernando Bombal en su artículo, un paseo por el infinito, refiriéndose a la percepción que tenía Aristóteles sobre el infinito, dice:

“...cualquier intervalo, espacial o temporal, parece poder dividirse indefinidamente..

Aristóteles se percató de éstos hechos y, como es imposible negar la existencia de procesos que parecen no tener fin, utilizó su sempiterno sentido común para separar el concepto de infinito en dos: y así introdujo la noción de infinito potencial, para designar magnitudes o procesos que se pueden prolongar tanto como se desee, en oposición al infinito actual o real" [2].

En el mismo texto, líneas más adelante dice el autor que Aristóteles acepta que existen magnitudes que pueden dividirse de forma indefinida, el espacio y el tiempo por ejemplo, o pueden aumentar indefinidamente como es el caso de los números naturales, pero rechaza la existencia de conjuntos infinitos como un todo.

\section{EL INFINITO POTENCIAL}

Es Aristóteles quien a pesar de su negativa con la idea del infinito, lo caracteriza en dos clases, el infinito potencial y el infinito actual. El infinito potencial se refiere al contar, al uno más, al siguiente de. El método de inducción matemática es una forma del infinito potencial. Se prueba para un elemento del conjunto (proceso de verificación), se supone verdadero para un elemento $k$ del conjunto y finalmente se prueba para el siguiente elemento, es decir, para $k+1$, pero $k$ puede ser un elemento tan grande como pueda caber en la imaginación del lector, entonces $k+1$ irá un paso más allá a lo que pueda imaginar cualquier lector. Analicemos un ejemplo del infinito potencial aplicado a la inducción matemática:

Ejemplo: demostremos que $n ! \geq n^{2}$ para $\forall n \in \mathbb{N}_{,} n \geq 4$

Primero: Se prueba para algún elemento del conjunto (verificación), para éste caso tomaremos $n=5$, con lo que se tiene que $5 ! \geq 5^{2}$, es decir, $120 \geq 25$, lo que verifica la propiedad.

Segundo: Suponemos un elemento $k$ (hipótesis inductiva), lo que nos queda $k ! \geq k^{2}$, se supone que es verdadera la hipótesis inductiva.

Tercero: Finalmente debemos verificar que se cumple para un elemento cualquiera del conjunto $(k+1) ! \geq(k+1)^{2}$, esta última expresión es la tesis, que tiene un significado particular si se mira con detenimiento. $k$, es un elemento cualquiera de un conjunto que es infinito, pero $k$ no es el último elemento del conjunto, porque de ser así, el conjunto sería finito, pero se demuestra para $k+1$, es decir, para un elemento que está después de cualquier elemento del conjunto, en otras palabras, se demuestra para cualquier elemento que se tome de colección infinita, en éste caso infinitamente grande. Ahora bien, partiendo de la hipótesis inductiva, se tiene que:

$k ! \geq k^{2}$, si multiplicamos en ambos lados de la desigualdad por $k+1$, que es un número positivo, pues $k$ es un número natural, se obtiene que $k !(k+1) \geq(k+1) k^{2} \quad$ luego $(k+1) ! \geq k^{a}+k^{2}$, cuidado que viene el artificio, es necesario sumar y restar una cantidad, de tal manera que nos podamos apoyar en la hipótesis inductiva, entonces

$(k+1) ! \geq k^{\mathrm{a}}+k^{2}+2 k-2 k+1-1$, lo que se hizo fue sumar y restar $2 k+1$, es decir, un número infinitamente grande, o en otras palabras, un número tan grande como se quiera. Finalmente se obtiene:

$$
(k+1) ! \geq\left(k^{2}+2 k+1\right)+\left(k^{3}-2 k-1\right)
$$


$(k+1) ! \geq(k+1)^{2}+\left(k^{a}-2 k-1\right) Y$

$(k+1) ! \geq(k+1)^{2}$, con lo que queda probada la

Proposición. Es importante notar que

$\left(k^{a}-2 k-1\right)>0$, para $k \geq 4$.

Lo que se acaba de probar es que el factorial de cualquier número natural mayor que 4 es mayor que el número elevado al cuadrado. Podemos tomar un número natural muy alejado del cero y la proposición anterior se cumple, ¿qué tan alejado? Tanto como se quiera.

Otra forma del infinito potencial se evidencia en el análisis matemático, con la propiedad arquimediana. El infinito potencial es un contar que se regula bajo un principio aquimediano [3], y dicho de esta manera parece un asunto que en nada atenta contra el sentido común, pero observando el teorema y su demostración puede ser que se cambie de opinión.

Teorema (propiedad arquimediana): para todo par de elementos $x, y \in \mathbb{R}$, con $x>0$, existe un número $n \in \mathbb{N}$ tal que $n x>y$.

Lo que dice intuitivamente el teorema, es que dado un número real cualquiera, existe un número natural que lo supera, es decir, si el lector se imagina un número real inmensamente grande siempre habrá un número natural que será mucho más grande que el número que cupo en la mente del lector. Ahora bien, la prueba del enunciado anterior no es tan intuitiva, hay que recurrir al razonamiento por el absurdo.

\section{Prueba (razonando por el absurdo)}

Sean $x_{s} y \in \mathbb{R}$, y $x>0$. Supongamos que $\forall n \in \mathbb{N}$. $n x \leq y$. Es necesario analizar dos casos. El primer caso se da si $y \leq 0$ entonces $x \leq 0$ lo que contradice la hipótesis, pues $x>0$. El segundo caso que se analiza es $y>0$, tomamos el conjunto $A=\{n x: n \in \mathbb{N}\}$, donde $A \subseteq \mathbb{R}$, es claro que $A \neq \emptyset$, pues $x \in A$. Además $A$ está acotado superiormente por $y$, es decir, en virtud del axioma del supremo $\alpha=\sup A$, como $x>0$ entonces $-x<0$ y $\alpha-x<\alpha$ lo que muestra que $\alpha-x$ no es una cota superior de $A$, como consecuencia de lo anterior debe existir $a_{0} \in A$ tal que $a-x<a_{0}$, esto es,

$\exists n_{0} \in \mathbb{N}$, entonces $a-x<x n_{0}=a_{0}$ y $\alpha<x\left(n_{0}+1\right)$, pero $\left(n_{0}+1\right) \in A$, lo que contradice la definición del supremo. [4].

Esta es una demostración clásica de la propiedad arquimediana que llevada atada a sus entrañas lo más oscuro y pequeño del infinito potencial. Entonces podría el lector preguntarse, ¿por qué es necesario construir una demostración tan elaborada para probar algo que parece tan obvio? Y la respuesta es clara, estamos hablando del infinito potencial, del infinito de las cantidades infinitamente pequeñas, o infinitamente grandes ¿qué tan pequeñas? Tanto como su imaginario se lo permita. En otras palabras, podríamos afirmar que el infinito potencial es la esencia del análisis matemático. O tal vez, tomando prestadas las palabras de David Berlinski, en su texto ascenso infinito, sería más acertado afirmar que hay un lugar en las matemáticas en el que se agrupan las ideas dudosas y desviadas. Están los infinitesimales por ejemplo. Son números menores que cualquier otro número pero sin llegar a cero [5].

\section{EL INFINITO ACTUAL}

Fue el infinito actual, el culpable de la negativa de Gauss, "protesto contra el uso del infinito como una entidad actual; ésta nunca se puede permitir en matemáticas. El infinito es sólo una forma de hablar cuando en realidad deberíamos hablar de límites a los cuales ciertas razones pueden aproximarse tanto como se desee, mientras otras son permitidas crecer limitadamente" [6].

El infinito actual originado en un contexto geométrico, es un infinito ilimitado, métrico, que permite la cuantificación y la resolución de problemas del mundo real [7]. Dice J. de Lorenzo, en el artículo, El infinito matemático.

Es el infinito actual, el que deja sin sustento el axioma euclidiano que afirma que el todo es mayor que las partes. Pues a partir de una biyección o una correspondencia uno a uno se puede demostrar que la cardinalidad de un conjunto es igual a la de un subconjunto propio, jel todo no siempre es mayor que las partes! Pero ¿qué significa semejante afirmación? Simplemente que cuando se hace referencia a los infinitos éstos parecen tener vida propia y comportarse a su antojo y lo que parece natural e intuitivo tiene formas más profundas. Es importante resaltar que el principal obstáculo del infinito actual eran las paradojas que generaba. Aunque para algunos matemáticos, como Bernahrd Bolzano, esto no era ningún problema él lo acepto con mucha naturalidad, hizo una venia y continuó su camino en las matemáticas. Escribió un libro que tituló las paradojas del infinito y enfatizó en la importancia de tratar el concepto del infinito con más precisión, porque según el gran matemático, esto era lo que generaba las contradicciones. Fue la naturalidad frente al concepto, la precisión con la que definió la idea de infinito lo que le dio paso a la teoría actual de conjuntos. En la actualidad, agradeciendo a Bolzano, por supuesto, se diría que un conjunto $X$ tiene cardialidad $n$, con $n$ natural, si es posible establecer una biyección del conjunto $X$ con el conjunto $\{1,2,3, \ldots n\}$, y en cuanto a los conjuntos finitos decimos que un conjunto $X$ es finito si tiene cardinal $n$ y un conjunto es infinito si no existe una correspondencia biunívoca con ningún conjunto $\{1,2,3, \ldots, n\}$ para ningún natural $n$. Tal vez algunos lectores se estén preguntando por el conjunto vacío, ¿es finito o infinito? La respuesta parece ser obvia, es finito, ¿y la razón? Esta no es tan obvia, es la salida de algunos matemáticos a infinidad de preguntas incomodas, ipor definición! 
Uno de los legados del infinito actual es el trabajo que realiza Cantor en la teoría de conjuntos, donde es posible hacer afirmaciones poco creíbles pero demostrables, sólo por citar algunas:

Primero: El conjunto de los números naturales es un subconjunto propio de los racionales positivos, es decir, todo elemento que está en $\mathbb{N}$ también está en $\mathbb{Q}$, pero el número de elementos de $\mathbb{N}$ es el mismo de $\mathbb{Q}$. Un conjunto es subconjunto de otro, pero el número de elementos es el mismo. Esto se puede mostrar construyendo una tabla para establecer una biyección entre los naturales y los racionales positivos. Cualquier número racional positivo puede escribirse de la forma $\frac{a}{b}$, siendo $a$ y $b$ números naturales.

\begin{tabular}{|c|c|c|c|c|c|c|c|}
\hline$a / b$ & 1 & 2 & 3 & 4 & 5 & 6 & $x=$ \\
\hline 1 & $1 / 1(1)$ & $1 / 2(2)$ & $1 / 3(6)$ & $1 / 4(7)$ & $1 / 5$ & $1 / 6$ & $\mathrm{NDE}$ \\
\hline 2 & $2 / 1$ (3) & $2 / 2(5)$ & $2 / 3(8)$ & $2 / 4$ & $2 / 5$ & $2 / 6$ & $\mathrm{xax}$ \\
\hline 3 & $3 / 1(4)$ & $3 / 2(9)$ & $3 / 3$ & $3 / 4$ & $\sqrt[3]{5}$ & $3 / 6$ & $\mathrm{max}$ \\
\hline 4 & $4 / 1(10)$ & $\sqrt[4]{/ 2}(1)$ & $4 / 3$ & $4 / 4$ & $4 / 5$ & $4 / 6$ & $\mathrm{mx}$ \\
\hline$\vdots$ & (11) & $\vdots$ & $\vdots$ & $\vdots$ & & $\vdots$ & \\
\hline
\end{tabular}

En la tabla anterior se puede observar que todos los números racionales se pueden inscribir en la tabla y siempre podrán "emparejarse" con un número natural, luego la cardinalidad de los números naturales y los números racionales positivos es la misma. Si continuamos razonando de la misma manera, entonces podríamos preguntarnos si existen conjuntos infinitos mayores que otros, y de ser así, ¿cómo es posible demostrar que algo que es infinito es mayor que otra cantidad infinita? De nuevo hay que recurrir al discurso inventado por los griegos, la reducción al absurdo.

Segundo el conjunto de números reales es mayor que el conjunto de números racionales. En términos de cardinalidad.

Tomemos el intervalo $(0,1)$ que es un subconjunto de $\mathbb{R}$, es claro que cualquier número en éste intervalo puede escribirse de la forma.

$0 \leq 0, a_{1} a_{2} a_{2} a_{4 m} \leq 1$, con $0 \leq a_{n} \leq 9$ con $n \in \mathbb{N}$.

Supongamos que $\mathbb{R}$ tiene la misma cardinalidad que los racionales, esto quiere decir que los reales se pueden poner en biyección con los naturales y utilizando el método de la diagonal se pueden representar los reales entre 0 y 1 , de la siguiente forma:

$1 \rightarrow 0, a_{11} a_{12} a_{13} a_{14}$

$2 \rightarrow 0, a_{11} a_{12} a_{13} a_{14}$

$3 \rightarrow 0, a_{11} a_{12} a_{13} a_{14}$

$4 \rightarrow 0, a_{11} a_{12} a_{13} a_{14}$

$a_{i j}: j$-ésimo digito del $i$-ésimo número, lo que quiere decir que cualquier número entre $[0,1]$ ésta en la lista anterior. Ahora construyamos un número particular formado por las diagonales, este número es:

$D=0, d_{1} d_{2} d_{a \ldots}$
$d_{1}=a_{11}+1$
$d_{2}=a_{22}+1$
$d_{a}=a_{a 3}+1$

Con la salvedad de que si algún $d_{\mathrm{ii}}=9+1$ entonces $d_{\mathrm{i}}=10$, luego es necesario decir que $a_{\mathrm{ii}}<9$ y además $D=0, d_{1} d_{2} d_{3 \ldots} \quad 0<D \leq 1$, luego el número está en la lista, o debería estarlo, pero

$d_{1}=a_{11}+1 \neq a_{11}$
$d_{2}=a_{22}+1 \neq a_{22}$
$d_{a}=a_{2 a}+1 \neq a_{2 a}$
$\vdots$

Luego, $D$ no puede estar en la lista, lo que es un absurdo porque en la lista estaban todos los números reales entre cero y uno. En conclusión la cardinalidad de los reales es mayor que la cardinalidad de los números naturales pues anteriormente se había probado que los naturales y los racionales tenían la misma cardinalidad [8].

\section{CONCLUSIONES}

El infinito es un concepto que debe tratarse de una perspectiva racional, abandonando el sentido común.

Es importante diferenciar el infinito potencial del infinito actual, desde la perspectiva histórica y moderna.

El infinito potencial deja como legado el análisis matemático, el cálculo diferencial, las series...

El infinito actual deja como legado la teoría de conjunto bien estructurada y los transfinitos de Cantor

\section{REFERENCIAS}

[1] J. R. Ortiz "El concepto de infinito". Asociación Matemática Venezolana, Vol. I, No. 2, pp. 60, 1994.

[2] F. Bombal. "un paseo por el infinito", rev, Real Acad. Ci Exact. Fis. Nat. (Esp), vol 104, n² (2010)

[3] J. de Lorenzo. El infinito matemático. Investigación y ciencia, temas 23 , primer trimestre, pp 6, 2001

[4] R. G. Bartle "Introducción al análisis matemático de una variable" Limusa, México D.F. 2010, p.

[5] B. David. escenso infinito. Modern library, New York, 2005, p. 68.

[6] J. F. Ortiz. "El concepto de infinito". Asociación Matemática Venezolana, Vol. I, No. 2, pp. 63, 1994

[7] J. de Lorenzo. El infinito matemático. Investigación y ciencia, temas 23, primer trimestre pp 9, 2001

[8] Pedro Saretta. 03/11/2010. Los infinitos de cantor. Recuperado https://www.youtube.com/watch?v=f1Ak-6vMVpg 